\title{
COVID-19 e os riscos de contaminação por coronavírus do Cirurgião Dentista em
}

\section{atividade clínica}

\author{
COVID-19 and the risks of coronavirus contamination of the Dental Surgeon in clinical activity
}

COVID-19 y los riesgos de contaminación por coronavirus del Cirujano Dentista en la actividad clínica

Cléa Adas Saliba Garbin

ORCID: https://orcid.org/0000-0001-5069-8812 Universidade Estadual Paulista, Brasil

E-mail: clea.saliba-garbin@unesp.br Julio Martinez Alves Oliveira

ORCID: https://orcid.org/0000-0002-3173-9444 Universidade Estadual Paulista, Brasil E-mail: juliooliveira1994@hotmail.com

Ana Victória Butarelo

ORCID: https://orcid.org/0000-0003-1145-7607 Universidade Estadual Paulista, Brasil

E-mail: anavictoria.butarelo@gmail.com

Suzely Adas Saliba Moimaz

ORCID: https://orcid.org/0000-0002-4949-529X Universidade Estadual Paulista, Brasil

E-mail: suzely.moimaz@unesp.br

Tânia Adas Saliba

ORCID: https://orcid.org/0000-0003-1327-2913 Universidade Estadual Paulista, Brasil E-mail: tania.saliba@unesp.br

Artênio José Isper Garbin

ORCID: https://orcid.org/0000-0002-7017-8942 Universidade Estadual Paulista, Brasil

E-mail: artenio.garbin@unesp.br

\begin{abstract}
Resumo
O ano de 2020 será marcado no contexto histórico mundial como o ano do enfrentamento de uma pandemia causada por um inimigo invisível, o vírus transmissor do COVID-19. A doença foi descoberta em dezembro de 2019 e a transmissão por aerossóis é possível quando há uma grande concentração em ambientes relativamente fechados. $\mathrm{O}$ objetivo neste estudo foi analisar os protocolos de controle e prevenção e os riscos de contaminação por COVID- 19 na prática clínica odontológica. Trata-se de uma pesquisa documental de protocolos, recomendações, guias, manuais e orientações, sobre medidas de prevenção e controle da COVID-19, disponíveis nas principais bases de dados e páginas eletrônicas de órgãos governamentais e instituições da área da saúde nacionais e internacionais. Foram obtidos e incluídos na pesquisa, 7 documentos, dentre eles notas técnicas emitidas pela Organização Mundial da Saúde, Ministério da Saúde, Agência Nacional de Vigilância Sanitária (ANVISA) e Conselho Federal de Odontologia (CFO). Na análise de conteúdo dos documentos, foi construído um quadro para extração dos dados. Foram observadas as seguintes variáveis nos documentos analisados: uso de Equipamento de Proteção Individual (Máscaras de proteção, gorro, luvas, protetores faciais e jaleco), orientação de etiqueta respiratória, uso de colutório antimicrobiano, recomendação para uso de instrumentais manuais, esterilização adequada dos instrumentais. As regras de distanciamento entre profissionais e pacientes, higienização das mãos, limpeza e desinfecção do ambiente de trabalho constaram em todos os documentos. O conhecimento sobre as formas de contágio da COVID-19 e a adoção de medidas preventivas no atendimento de pacientes é importante para evitar a disseminação da doença.
\end{abstract}

Palavras-chave: COVID-19; Cirurgião Dentista; Prevenção.

\begin{abstract}
The year 2020 will be marked in the world historical context as the year of facing a pandemic caused by an invisible enemy, the virus that transmits COVID-19. It is a family of viruses that causes respiratory infections. The disease was discovered in December 2019 and aerosol transmission is possible when there is a high concentration in relatively closed environments. The aim of the study was to analyze the control and prevention protocols and the risks of contamination by COVID-19 in dental clinical practice. This is a documentary research of protocols, recommendations, guides, manuals and guidelines on COVID-19 prevention and control measures, available in the main databases and websites of government agencies and institutions in the national and international health area. .
\end{abstract}


Seven documents were obtained and included in the research, including technical notes issued by the World Health Organization, Ministry of Health, National Health Surveillance Agency (ANVISA) and Federal Council of Dentistry (CFO). In the analysis of the content of the documents, a framework for data extraction was built. The following variables were observed in the analyzed documents: use of Personal Protective Equipment (Protective masks, cap, gloves, face shields and lab coat), respiratory label guidance, use of antimicrobial mouthwash, recommendation for the use of manual instruments, adequate sterilization of the instrumentals. The rules of distance between professionals and patients, hand hygiene, cleaning and disinfection of the work environment were included in all documents. Knowledge about the ways in which COVID-19 is contagious and the adoption of preventive measures in patient care is important to prevent the spread of the disease.

Keywords: COVID-19; Dental Surgeon; Prevention.

\section{Resumen}

El año 2020 estará marcado en el contexto histórico mundial como el año de afrontar una pandemia provocada por un enemigo invisible, el virus que transmite COVID-19. La enfermedad se descubrió en diciembre de 2019 y la transmisión por aerosoles es posible cuando hay una alta concentración en entornos relativamente cerrados. El objetivo de este estudio fue analizar los protocolos de control y prevención y los riesgos de contaminación por COVID- 19 en la práctica clínica odontológica. Se trata de una investigación documental de protocolos, recomendaciones, guías, manuales y guías, sobre medidas de prevención y control de COVID-19, disponibles en las principales bases de datos y páginas electrónicas de organismos gubernamentales e instituciones de salud nacionales e internacionales. Se obtuvieron e incluyeron siete documentos en la investigación, entre ellos notas técnicas emitidas por la Organización Mundial de la Salud, el Ministerio de Salud, la Agencia Nacional de Vigilancia Sanitaria (ANVISA) y el Consejo Federal de Odontología (CFO). En el análisis del contenido de los documentos, se construyó un marco para la extracción de datos. En los documentos analizados se observaron las siguientes variables: uso de Equipo de Protección Personal (mascarillas protectoras, gorro, guantes, caretas y bata de laboratorio), orientación sobre etiqueta respiratoria, uso de enjuague bucal antimicrobiano, recomendación para el uso de instrumentos manuales, esterilización adecuada de instrumental. En todos los documentos se incluyeron las normas de distanciamiento entre profesionales y pacientes, higiene de manos, limpieza y desinfección del ambiente de trabajo. El conocimiento sobre las formas de contagio en COVID-19 y la adopción de medidas preventivas en la atención al paciente es importante para prevenir la propagación de la enfermedad.

Palabras clave: COVID-19; Cirujano Dentista; Prevención.

\section{Introdução}

O ano de 2020 será marcado no contexto histórico mundial como o ano do enfrentamento de uma pandemia causada por um inimigo invisível, o SARS-CoV-2, causador da COVID-19, pertencente à uma família de vírus que causa infecções respiratórias e foi descoberto em dezembro de 2019, na província de Wuhan, China.

Sabe- se que o COVID-19 se espalha principalmente por meio de gotículas. O consultório odontológico é um abiente com grande potencial de disseminação do vírus que pode ocorrer por aerossol durante procedimentos odontológicos, como o uso de peças de mão de alta e baixa velocidade, raspadores ultra-sônicos, seringas de ar / água, intra orais , radiografias ou com um paciente infectado tossindo (COVID-19 Dental Services Evidence Review (CoDER) Working Group, 2020).

A prevenção da infecção cruzada é aspecto crucial na prática odontológica. Durante o trabalho, é fundamental que sejam adotadas rotinas básicas de prevenção (Taiwo \& Aderinokun, 2002; Thomas, Jarboe \& Frazer, 2008). Estas ações minimizam os riscos de transmissão de doenças infectocontagiosas, protegendo pacientes, o ambiente de assistência odontológica e a equipe (Brasil, 2000; Ferreira, 1995; Garbin et al., 2005; Gonçalves, Travassos \& Silva, 1996; Medeiros, Cardoso \& Ferreira, 1998).

Por conta do alto risco de exposição à agentes biológicos, é extremamente importante que os cirurgiões-dentistas e equipe auxiliar estejam com os conhecimentos atualizados sobre biossegurança, visando reduzir o risco de contaminação cruzada, o que exige estudo, responsabilidade e muita disciplina e oferece maior segurança durante as atividades clínicas (Engelmann et al., 2010).

É necessário que haja a adoção de medidas que evitem a transmissão de microrganismos, tornando indispensável o conhecimento dos profissionais da saúde a respeito de medidas e práticas de biossegurança, reduzindo os riscos inerentes ao ambiente de atuação (Lopes et al., 2019). 
O uso correto do Equipamento de Proteção Individual (EPI), incluindo máscara, óculos de proteção, luva descartável e jaleco é de suma importância pois trata-se de uma barreira física eficiente de prevenir a contaminação e é obrigatório durante os atendimentos, limpeza do ambiente e processamento de produtos (Agência Nacional de Vigilância Sanitária, 2006).

A adoção de medidas de proteção deve ser preconizada. Durante a atividade clínica, a exposição ao vírus é aumentada com a permanência de gotículas de sangue e saliva no ar, em instrumentais, e equipamentos de proteção utilizados. A pandemia causada pelo COVID-19 resultou no fechamento de consultórios odontológicos ou na redução dos serviços em todo o mundo. Neste momento, alguns países estão reabrindo ou planejando reabrir clínicas odontológicas como parte de uma saída da estratégia de bloqueio (COVID-19 Dental Services Evidence Review (CoDER) Working Group, 2020).

No Brasil, diversas instituições e órgãos responsáveis pela vigilância sanitária e exercício da profissão divulgaram recomendações à respeito da reabertura dos serviços em resposta à necessidade urgente de orientação, tendo em vista o alto risco de contaminação durante a prática clínica odontológica. Sendo assim, esta revisão de protocolos foi realizada para auxiliar os cirurgiões-dentistas e proprietários de clínicas odontológicas para retorno às atividades.

O objetivo neste estudo foi analisar os protocolos de controle e prevenção e os riscos de contaminação do COVID-19 na prática clínica odontológica.

\section{Metodologia}

Trata-se de uma pesquisa documental de protocolos, recomendações, guias, manuais e orientações, sobre medidas de prevenção e controle da COVID-19, disponíveis nas principais bases de dados e páginas eletrônicas de órgãos governamentais e instituições da área da saúde nacionais e internacionais. O método científico é um trabalho sistemático, na busca de respostas às questões estudadas, é o caminho que se deve seguir para levar à formulação de uma teoria científica. (Pereira et al., 2018)

Foram obtidos e incluídos na pesquisa, 7 documentos, dentre eles notas técnicas emitidas pela Organização Mundial da Saúde, Ministério da Saúde, Agência Nacional de Vigilância Sanitária (ANVISA) e Conselho Federal de Odontologia (CFO). Na análise de conteúdo dos documentos, foi construído um quadro para extração dos dados.

Foram observadas as seguintes variáveis nos documentos analisados: uso de Equipamento de Proteção Individual (Máscaras de proteção, gorro, luvas, protetores faciais e jaleco), orientação de etiqueta respiratória, uso de colutório antimicrobiano, recomendação para uso de instrumentais manuais, e esterilização adequada dos instrumentais.

Foram analisados estudos relacionados ao risco de contaminação do cirurgião dentista durante o atendimento odontológico, a adoção de medidas de biossegurança recomendadas e os cuidados necessários após a ida ao consultório odontológico.

Durante a pesquisa foram utilizados dados secundários disponíveis em sites oficiais das principais bases de dados encontrados sobre o tema, sem identificação de sujeitos, sendo dispensada de apreciação em comitê de ética em pesquisa, em conformidade com a Resolução 466/2012 do Conselho Nacional de Saúde.

\section{Resultados}

Para o desenvolvimento da pesquisa, foram consultadas as principais bases de dados relacionadas à saúde além de órgãos responsáveis pela determinação de normas de trabalho aos profissionais de saúde (ANVISA, Ministério da Saúde, OMS) e aos cirurgiões dentistas (CFO). Estas informações podem ser visualizadas na Tabela 1. 
Tabela 1. Manuais, protocolos e documentos orientadores sobre Biossegurança nos serviços de saúde, em especial no atendimento Odontológico, em tempos de pandemia do COVID-19. Brasil, 2020.

\begin{tabular}{|c|c|c|c|}
\hline Nome do Documento & Fonte & Instituição & $\begin{array}{l}\text { Data de } \\
\text { Divulgação }\end{array}$ \\
\hline $\begin{array}{l}\text { Manual de Boas Práticas em Biossegurança Para } \\
\text { Ambientes Odontológicos } 2020 \text {. }\end{array}$ & $\begin{array}{l}\text { Conselho Federal de } \\
\text { Odontologia }\end{array}$ & $\begin{array}{lll}\text { Conselho } & \text { Federal de } \\
\text { Odontologia } & \end{array}$ & $20 / 04 / 2020$ \\
\hline $\begin{array}{l}\text { Guia Odontológico para atendimento durante a } \\
\text { pandemia }\end{array}$ & Unesp Araraquara & $\begin{array}{l}\text { Universidade Estadual Paulista } \\
\text { Júlio de Mesquita Filho }\end{array}$ & 2020 \\
\hline $\begin{array}{l}\text { Protocolos e orientações aos profissionais e serviços } \\
\text { de saúde sobre atendimentos - COVID-19 }\end{array}$ & Ministério da Saúde & Governo Federal & $23 / 03 / 2020$ \\
\hline $\begin{array}{l}\text { Orientações para serviços de saúde:Medidas de } \\
\text { Prevenção e controle que devem ser adotadas durante } \\
\text { a assistência aos casos suspeitos ou confirmados de } \\
\text { infecção pelo novo coronavírus (SARS-COV-2) }\end{array}$ & $\begin{array}{lr}\text { Anvisa- } & \text { Agência } \\
\text { Nacional } & \text { de } \\
\text { Vigilância } & \text { Sanitária }\end{array}$ & $\begin{array}{l}\text { Ministério da Saúde-Governo } \\
\text { Federal }\end{array}$ & $31 / 03 / 2020$ \\
\hline $\begin{array}{l}\text { Orientações de biossegurança laboratorial relativa a } \\
\text { doença do coronavírus } \\
\text { (COVID19):orientação } \\
\text { provisória }\end{array}$ & PAHO/OMS & $\begin{array}{l}\text { Organização Pan- Americana de } \\
\text { Saúde/ Organização Mundial da } \\
\text { Saúde }\end{array}$ & $19 / 03 / 2020$ \\
\hline $\begin{array}{l}\text { Consenso ABENO: Biossegurança no ensino } \\
\text { odontológico pós-pandemia da COVID-19 }\end{array}$ & $\begin{array}{l}\text { Abeno: Associação } \\
\text { Brasileira de Ensino } \\
\text { Odontológico }\end{array}$ & $\begin{array}{l}\text { Abeno: Associação Brasileira } \\
\text { de Ensino Odontológico }\end{array}$ & $03 / 07 / 2020$ \\
\hline $\begin{array}{l}\text { Recommendations for the re-opening of dental } \\
\text { services: a rapid review of international sources }\end{array}$ & $\begin{array}{l}\text { COVID-19 Dental } \\
\text { Services Evidence } \\
\text { Review (CoDER) } \\
\text { Working Group }\end{array}$ & $\begin{array}{l}\text {-NHS: Education for Scotland } \\
\text { - Cochrane Oral Health } \\
\text { - Health services research unit } \\
\text {-SDCEP } \\
\text {-University of Aberdeen } \\
\text {-University of Dundee } \\
\text {-The University of Manchester }\end{array}$ & $06 / 05 / 2020$ \\
\hline
\end{tabular}

Fonte: Autores, Brasil (2021).

A rota de transmissão do coronavírus no consultório odontológico se dá através do transporte de gotículas e aerossóis contaminados pelo ar, que podem se depositar sobre uma superfície inanimada e através do contato direto ou indireto com o vírus pode resultar na contaminação do cirurgião-dentista, equipe auxiliar e pacientes.

A Tabela 2 mostra as principais formas de prevenir a contaminação pelo coronavírus durante o atendimento odontológico, de acordo com o CFO e ANVISA. 
Tabela 2. Principais formas de prevenção durante atendimento odontológico de acordo com o Conselho Federal de Odontologia (CFO) e Agência Nacional de Vigilância Sanitária (ANVISA).

\begin{tabular}{|c|c|c|c|}
\hline VARIÁVEIS & CFO & ANVISA & ABENO \\
\hline $\begin{array}{l}\text { Uso de equipamento de } \\
\text { proteção individual }\end{array}$ & $\begin{array}{l}\text { Jaleco impermeável, touca, } \\
\text { luvas, máscara cirúrgica, } \\
\text { protetor de superfícies, óculos e } \\
\text { face shield }\end{array}$ & $\begin{array}{l}\text { Gorro, óculos de proteção, } \\
\text { máscara N95/PFF2, face } \\
\text { shield, avental impermeável } \\
\text { e luvas de procedimento }\end{array}$ & $\begin{array}{l}\text { Avental descartável, gorro, touca, } \\
\text { Máscara cirúrgica tripla descartável } \\
\text { (tipo IIR) ou N95 ou PFF2, óculos de } \\
\text { proteção, pijama cirúrgico de mangas } \\
\text { curtas, face shield, sobreluvas, calçado } \\
\text { cirúrgico fechado e emborrachado }\end{array}$ \\
\hline $\begin{array}{l}\text { Orientação de etiqueta } \\
\text { respiratória }\end{array}$ & Sim & Sim & Sim \\
\hline $\begin{array}{l}\text { Regras de distanciamento } \\
\text { profissional-paciente }\end{array}$ & Sim & Sim & Sim \\
\hline Higienização das mãos & $\begin{array}{l}\text { Sabonete ou álcool } 70 \% \text { antes e } \\
\text { após contato com paciente }\end{array}$ & $\begin{array}{l}\text { Sabonete líquido ou álcool } \\
70 \%\end{array}$ & Sabonete líquido e/ou álcool 70\% \\
\hline $\begin{array}{l}\text { Uso de colutório } \\
\text { antimicrobiano }\end{array}$ & N/A & $\begin{array}{l}\text { Peróxido de hidrogênio de } \\
0,5 \text { a } 1 \% \text { ou polvidona a } \\
0,2 \% \text { ( } 15 \mathrm{ml} 30 \text { segundos } \\
\text { antes de iniciar a consulta) }\end{array}$ & $\begin{array}{l}15 \mathrm{ml} \text { de gluconato de clorexidina a } \\
0,12 \% \text { sem álcool ou de cloreto de } \\
\text { cetilpiridínio a } 1: 4.000 \text { ou } \\
\text { Iodopovidona a } 0,2 \% \text { para bochecho } \\
\text { durante } 1 \text { minuto }\end{array}$ \\
\hline $\begin{array}{l}\text { Limpeza e desinfecção do } \\
\text { ambiente de trabalho }\end{array}$ & Após cada atendimento & Após cada atendimento & $\begin{array}{l}30 \text { minutos após o término dos } \\
\text { atendimentos }\end{array}$ \\
\hline $\begin{array}{l}\text { Uso de instrumentais } \\
\text { manuais }\end{array}$ & N/A & Sempre que possível & Sempre que possível \\
\hline $\begin{array}{l}\text { Esterilização adequada dos } \\
\text { instrumentais }\end{array}$ & Sim & Sim & Sim \\
\hline
\end{tabular}

Fonte: Conselho Federal de Odontologia (2020). Agência Nacional de Vigilância Sanitária 2020. Associação Brasileira de ensino Odontológico (ABENO) (2020).

O ambiente é apontado como importante reservatório de microrganismos nos serviços de saúde. Assim, o Serviço de Limpeza e Desinfecção de Superfícies em Serviços de Saúde apresenta relevante papel na prevenção das infecções (Anvisa, 2010). A Tabela 3 mostra um quadro comparativo de desinfetantes de superfícies.

Tabela 3. Quadro comparativo de desinfetantes de superfícies.

\begin{tabular}{l|l|l}
\hline Desinfetante & Álcool a 70\% & Hipoclorito de sódio a 1\% \\
\hline Nível & Médio & Médio \\
\hline Aplicação & $\begin{array}{l}\text { Fricção em 3 etapas intercaladas pelo tempo de } \\
\text { secagem natural, totalizando 10 minutos }\end{array}$ & Aplicação na superfície por 2 a minutos \\
\hline Vantagens & $\begin{array}{l}\text { Fácil aplicação, ação rápida, compatível com } \\
\text { artigos metálicos, superfícies e tubetes } \\
\text { anestésicos }\end{array}$ & $\begin{array}{l}\text { Ação rápida, indicado para superfícies e } \\
\text { artigos não metálicos e materiais } \\
\text { termossensíveis }\end{array}$ \\
\hline Desvantagens & $\begin{array}{l}\text { Volátil, inativado por matérias orgânicas, } \\
\text { inflamável, resseca plásticos e opacifica acrílico }\end{array}$ & $\begin{array}{l}\text { Instável, corrosivo, inativado por } \\
\text { matérias orgânicas, irritação de pele e } \\
\text { mucosas }\end{array}$ \\
\hline
\end{tabular}

Fonte: Associação Brasileira de Ensino Odontológico (ABENO) (2020). 
Em resposta à necessidade urgente de orientação sobre a biossegurança em atendimentos odontológicos, diversos órgãos internacionais de saúde como os da França, buscaram auxiliar as autoridades com a produção de manuais de recomendações para pacientes, funcionários e Cirurgiões dentistas durante o atendimento clínico (COVID-19 Dental Services Evidence Review (CoDER) Working Group, 2020).

Tabela 4. Recomendações e Orientações de biossegurança durante atendimento clínico para Cirurgião Dentista, equipe de funcionários e pacientes na França.

\begin{tabular}{|c|c|c|}
\hline $\begin{array}{l}\text { Considerações sobre a recepção do paciente e área } \\
\text { de espera: }\end{array}$ & $\begin{array}{lrlll}\text { EPI para a equipe } & \text { de } \\
\text { funcionários }\end{array}$ & $\begin{array}{l}\text { Pós-operatório: limpeza } \\
\text { desinfecção / resíduos }\end{array}$ \\
\hline $\begin{array}{l}\text { - Pacientes devem usar máscaras } \\
\text { - Máscaras devem ser disponibilizadas, se necessário } \\
\text { - Telas de proteção para recepção } \\
\text { - Limite o uso da área de espera. } \\
\text { - Desinfetante para as mãos disponível. } \\
\text { Triagem de pacientes em grupos: } \\
\text { - Grupo A: "Pacientes saudáveis" todos tratamentos } \\
\text { disponíveis. } \\
\text { - Grupo B: Pacientes protegidos (mais } \\
65 \text { anos, cardíaco ou respiratório } \\
\text { doença, imunossuprimido) } \\
\text { - Grupo C: COVID-19 de alto risco, } \\
\text { pacientes que tiveram contato próximo } \\
\text { com COVID-19 confirmado: Tratamento de } \\
\text { emergência em } \\
\text { slots dedicados, atraso de trabalho não urgente (revisão } \\
\text { de } 14 \text { dias } \\
\text { incubação). } \\
\text { - Grupo D: COVID-19 confirmado } \\
\text { tratamento de emergência } \\
\text { em slots dedicados. Atrase o tratamento não urgente } \\
\text { (15 dias } \\
\text { após o início dos sintomas ou } 24 \\
\text { dias se imunossuprimido. } \\
\text { - Sem teste de sorologia ou diagnóstico - fazer o teste } \\
\text { na prática odontológica }\end{array}$ & $\begin{array}{l}\text { A equipe não- clínica deve usar } \\
\text { máscara cirúrgica em todos os } \\
\text { momentos e nunca entrar na sala } \\
\text { clínica } \\
\text { EPI; } \\
\text { - Aventais } \\
\text { - Protetor ocular } \\
\text { - Chapéu Cirúrgico } \\
\text { - Máscaras FFP2 } \\
\text { - Luvas descartáveis } \\
\text { - Bata cirúrgica extra adicional para } \\
\text { PPE }\end{array}$ & $\begin{array}{l}\text { Eliminação de resíduos clínicos: } \\
\text { Acesso imediato a bandeja de } \\
\text { desinfecção com tampa e } \\
\text { recipiente de resíduos clínicos } \\
\text { na sala de tratamento. } \\
\text { Os itens de uso único devem ser } \\
\text { destruídos após o uso. } \\
\text { Todos os resíduos clínicos e } \\
\text { EPIs devem ser eliminados. }\end{array}$ \\
\hline
\end{tabular}

Fonte: Ordem Nacional dos Cirurgiões-dentistas, França (2020).

É extremamente necessário que os cuidados sejam estendidos após um dia de trabalho, ao chegar em casa, evitando a contaminação do cirurgião-dentista e dos que moram em sua residência. A recomendação é que a rotina seja seguida rigorosamente de modo que ao voltar para casa o indivíduo não toque em nada sem antes se higienizar. Os sapatos devem ser retirados na área externa e a roupa lavada com alvejante acima de $60^{\circ}$. O celular, óculos e acessórios pessoais higienizados com álcool $70^{\circ}$. As áreas mais expostas como mãos, punhos, pescoços e rosto devem ser bem higienizadas durante o banho (Conselho Federal de Odontologia, 2020).

\section{Discussão}

Por meio de gotículas, a disseminação dos vírus como o coronavírus, podem percorrer mais de um metro e meio de distância (Kutter et al., 2018) e sobreviver nas superfícies inanimadas por alguns dias, principalmente quando são transportados por meio de secreção humana (Otter, Yezli, Salkeld \& French, 2013). O contato das mãos com superfícies contaminadas pode resultar na aquisição e transferência de patógenos para os olhos, nariz ou boca, ocasionando a contaminação e desenvolvimento da doença (Otter, Yezli, Salkeld \& French, 2013). A rota de contaminação no ambiente odontológico se dá através do 
transporte de gotículas contaminadas pelo vírus no ar, potencializando o risco de infecção direta ou indireta ao cirurgiãodentista, equipe auxiliar e pacientes (Kutter et al., 2018).

As medidas preventivas apresentam importantes resultados no combate a contaminação por coronavírus durante o atendimento clínico. O uso de Equipamentos de Proteção Individual como jaleco, máscara de proteção, gorros, óculos e luvas deve ser preconizado, de acordo com as orientações expostas pelos órgãos de vigilância sanitária e protocolos internacionais de recomendações para retorno de atividades clínicas (Anvisa, 2006; Conselho Federal de Odontologia, 2020; COVID-19 Dental Services Evidence Review (CoDER) Working Group, 2020).

É de suma importância que entre cada atendimento realize-se a higienização da cadeira odontológica, peças de mão e seringa tríplice, que são essenciais para o desenvolvimento do trabalho no ambiente odontológico, além da correta esterilização de instrumentais e pontas diamantadas já que estão em contato direto com a cavidade oral e os fluídos que são gerados durante o atendimento (Anvisa, 2006; Conselho Federal de Odontologia, 2020).

A França é um dos países Europeus que mais sofreu impacto com a nova doença, chegando a aproximadamente 3.239.256 casos da doença, registrando 77.209 mortes (Google Notícias, 2021). Diante dessa situação, foi de suma importância a reorganização dos serviços oferecidos à população, sobretudo serviços de saúde, incluindo a atenção odontológica, buscando evitar a disseminação da doença (COVID-19 Dental Services Evidence Review (CoDER) Working Group, 2020).

Sendo assim, visando evitar a contaminação do lar e dos que ali residem, o profissional, equipe auxiliar e pacientes devem tomar medidas preventivas quando chegam em casa, realizando a correta higienização das mãos, além da desinfecção de roupas, sapatos e pertences pessoais de maneira eficaz (Anvisa, 2006).

\section{Conclusão}

Os protocolos disponíveis para prevenção da transmissão do coronavírus mostram que é extremamente necessário o uso correto de EPI, incluindo face shield, avental impermeável e máscara N95/PFF2, além do distanciamento social, etiqueta respiratória, distanciamento entre profissional e paciente, limpeza e desinfecção do ambiente de trabalho e esterilização correta dos materiais. O cirurgião-dentista está constantemente exposto à contaminação pelo COVID-19, devido à proximidade com o paciente e a geração de aerossóis contaminados por fluídos salivares durante o atendimento, sendo necessário também que sejam tomadas medidas de controle e prevenção após os atendimentos, dando atenção ao correto manuseio de roupas e sapatos ao chegar em casa, além da higienização correta do celular e áreas mais expostas. Neste trabalho, foram consultados dados secundários, o que restringe análises mais aprofundadas dos protocolos. Embora o estudo apresente esta limitação, ele traz à tona dados importantes para discussão sobre a adoção de normas preventivas pelo cirurgião durante as atividades clínicas em tempos de pandemia da COVID-19. Novos estudos podem ser realizados visando verificar a atualização dos protocolos de atendimento para evitar a disseminação do COVID-19 ao longo do tempo, além de pesquisas tipo inquérito com profissionais da Odontologia, como cirurgiões-dentistas e equipe auxiliar, para verificar a segurança dos mesmos em relação ao risco de contaminação no ambiente de trabalho e os protocolos de biossegurança efetuados antes, durante e após cada atendimento.

\section{Agradecimentos}

O presente trabalho foi realizado com apoio da Coordenação de Aperfeiçoamento de Pessoal de Nível Superior - Brasil (CAPES) - Código de Financiamento 001.

\section{Referências}

Agência Nacional de Vigilância Sanitária. (2006). Serviços Odontológicos: prevenção e controle de riscos. Anvisa.

Agência Nacional de Vigilância Sanitária. (2010). Segurança do paciente em serviços de saúde: limpeza e desinfecção de superfícies. Anvisa. 
Research, Society and Development, v. 10, n. 10, e372101018540, 2021

(CC BY 4.0) | ISSN 2525-3409 | DOI: http://dx.doi.org/10.33448/rsd-v10i10.18540

Agência Nacional de Vigilância Sanitária. (2020). Nota técnica nº 04/2020 GVIMS/GGTES/ANVISA. https://www20.anvisa.gov.br/segurancadopaciente/ind ex.php/alertas/item/nota-tecnica-n-04-2020-gvims-ggtes-anvisa-atualizada?category_id=244

Associação Brasileira de Ensino Odontológico. (2020). Consenso ABENO: biossegurança no ensino odontológico pós pandemia da COVID-19. 2020. http://www.abeno.org.br/arquivos/downloads/retomada_de_praticas_seguras_no_ensino_odontologico.pdf

Brasil. (2000). Controle de infecção e prática odontológica em tempos de AIDS: manual de condutas. Ministério da Saúde.

Brasil. (2020). Protocolos e orientações aos profissionais e serviços de saúde sobre atendimentos - Covid19. https://www.conasems.org.br/covid-19protocolos-e-orientacoes-aos-profissionais-e-servicos-de-saude/

Conselho Federal de Odontologia. (2020). Manual de boas práticas em biossegurança para ambientes odontológicos. https://website.cfo.org.br/wpcontent/uploads/2020/04/cfo-lanc\%CC\%A7a-Manual-de-Boas-Pra\%CC\%81ticas-em-Biosseguranc\%CC\%A7a-para-Ambientes-Odontologicos.pdf

COVID-19 Dental Services Evidence Review (CoDER) Working Group. (2020). Recommendations for the re-opening of dental services: a rapid review of international sources. https://oralhealth.cochrane.org/sites/oralhealth.cochrane.org/files/public/uploads/covid19_dental_reopening_rapid_review_13052020.pdf

Engelmann, A. I., Daí, A. A., Miura, C. S. N., Bremm, L. L. \& Boleta-Ceranto, D. C. F. (2010). Avaliação dos procedimentos realizados por cirurgiõesdentistas da região de Cascavel-PR visando ao controle da biossegurança. Odontologia Clínico-Científica, 9(2), 161-165.

Ferreira, R. A. (1995). Barrando o invisível. Revista da Associação Paulista de Cirurgiões Dentistas, 49(6), 417-427.

Garbin, A. J. I., Garbin, C. A. S., Arcieri, R. M., Crossato, M. \& Ferreira, N. F. (2005). Biosecurity in public and private office. Journal of Applied Oral Science, 13(2), 163-166.

Gonçalves, A. C. S., Travassos, D. V. \& Silva, M. (1996). Biossegurança do exercício da odontologia. RPG: Revista de Pós-Graduação, 3(3), $242-245$.

Google notícias. (2021). Número de casos e mortes por Covid-19 nos países ao redor do mundo. Recuperado de https://news.google.com/covid19/map?hl=pt$\mathrm{BR} \& \mathrm{mid}=\% 2 \mathrm{Fm} \% 2 \mathrm{~F} 0 \mathrm{f} 819 \mathrm{c} \& \mathrm{gl}=\mathrm{BR} \& \mathrm{ceid}=\mathrm{BR} \% 3 \mathrm{Apt}-419$

Kutter, J. S., Spronken, M. I., Fraaij, P. L., Fouchier, R. A. \& Herfst, S. (2018). Transmission routes of respiratory viruses among humans. Current Opinion in Virology, 28, 142-151.

Lopes, A. L., Rodrigues, L. G., Zina, L. G., Palmier, A. C., Ferreira, F. G., Abreu, M. H. N. G. \& Vasconcelos, M. (2019). Biossegurança em odontologia: conduta dos estudantes antes e após uma ação educativa. Revista da ABENO, 19(2), 43-53.

Medeiros, U. V., Cardoso, A. S. \& Ferreira, S. M. S. (1998). Uso das normas de controle de infecção na prática odontológica. Revista Brasileira de Odontologia, 55, 4, 209-215.

Organização Pan-Americana da Saúde. (2021). Folha informativa covid19 (Doença causada pelo novo coronavírus). https://www.paho.org/bra/index.php?option=com_content\&view=article\&id=6101:covid19\&Itemid=875\#recomendacoes

Otter, J. A., Yezli, S., Salkeld, J. A. \& French, G. L. (2013). Evidence that contaminated surfaces contribute to the transmission of hospital pathogens and an overview of strategies to address contaminated surfaces in hospital settings. American Journal of Infection Control, 41(5 Suppl), S6-S11.

Pereira A. S. et al. (2018). Metodologia da pesquisa científica. UFSM. https://repositorio.ufsm.br/bitstream/handle/1/15824/Lic_Computacao_MetodologiaPesquisa-Cientifica.pdf?sequence $=1$.

Ricci, W.A. (2020). Guia odontológico para atendimento durante a pandemia Covid 19: manual odontológico de biossegurança COVID19. Araraquara: Unesp.

Taiwo, J. O. \& Aderinokun, G. A. (2002). Assessing cross infection prevention measures at the Dental Clinic, University College Hospital, Ibadan. African Journal of Medicine and Medical Sciences, 31(3), 213-217.

Thomas, M. V., Jarboe, G. \& Frazer, R. Q. (2008). Infection control in the dental office. Dental Clinics of North America, 52(3), 609-628. 